\title{
Supporting Long-term Reproducible Software Execution
}

\author{
Luís Oliveira \\ loliveira@pitt.edu \\ University of Pittsburgh \\ Daniel Mossé \\ mosse@cs.pitt.edu \\ University of Pittsburgh
}

\author{
David Wilkinson \\ dwilk@cs.pitt.edu \\ University of Pittsburgh \\ Bruce Childers \\ childers@cs.pitt.edu \\ University of Pittsburgh
}

\begin{abstract}
A recent widespread realization that software experiments are not as easily replicated as once believed brought software execution preservation to the science spotlight. As a result, scientists, institutions, and funding agencies have recently been pushing for the development of methodologies and tools that preserve software artifacts. Despite current efforts, long term reproducibility still eludes us.

In this paper, we present the requirements for software execution preservation and discuss how to improve long-term reproducibility in science. In particular, we discuss the reasons why preserving binaries and pre-built execution environments is not enough and why preserving the ability to replicate results is not the same as preserving software for reproducible science. Finally, we show how these requirements are supported by Occam, an open curation framework that fully preserves software and its dependencies from source to execution, promoting transparency, longevity, and re-use. Specifically, Occam provides the ability to automatically deploy workflows in a fully-functional environment that is able to not only run them, but make them easily replicable.
\end{abstract}

\section{CCS CONCEPTS}

- Applied computing $\rightarrow$ Digital libraries and archives; $\bullet$ Software and its engineering $\rightarrow$ Reusability; Software verification and validation;

ACM Reference Format:

Luís Oliveira, David Wilkinson, Daniel Mossé, and Bruce Childers. 2018. Supporting Long-term Reproducible Software Execution. In P-RECS'18: First International Workshop on Practical Reproducible Evaluation of Computer Systems, fune 11, 2018, Tempe, AZ, USA. ACM, New York, NY, USA, 6 pages. https://doi.org/10.1145/3214239.3214245

\section{INTRODUCTION}

In recent years scientists have been acknowledging the existence of a reproducibility crisis, because scientists from many different fields now realize that their software experiments are not as reproducible

Permission to make digital or hard copies of all or part of this work for personal or classroom use is granted without fee provided that copies are not made or distributed for profit or commercial advantage and that copies bear this notice and the full citation on the first page. Copyrights for components of this work owned by others than the author(s) must be honored. Abstracting with credit is permitted. To copy otherwise, or republish, to post on servers or to redistribute to lists, requires prior specific permission and/or a fee. Request permissions from permissions@acm.org.

P-RECS'18, fune 11, 2018, Tempe, AZ, USA

(C) 2018 Copyright held by the owner/author(s). Publication rights licensed to ACM. ACM ISBN 978-1-4503-5861-3/18/06 ..\$15.00

https://doi.org/10.1145/3214239.3214245 as they once thought they were [3] (a tenet of science!!). Meanwhile, computational artifacts become more and more ubiquitous, and essential, for scientific progress [19].

As a reaction to this reproducibility crisis, many scientists started to develop their own guidelines and methodologies $[1,5,20]$ to improve the ability to reproduce their experiments. Simultaneously, other scientists started to develop tools to improve the reproducibility of software experiments, ranging from the simplest creation of a virtual machine containing their computational artifacts, to packaging systems containing all the binaries required to run the software used in the experiments. The consequence is that, where once only a few systems addressed experimental result reproduction, now a large number of tools try to address specific issues, in a fragmented and incomplete effort [21].

Unfortunately, available tools take a simplified route to implement their solutions. Some, only preserve the binaries strictly required to repeat the same exact experiment. Others, preserve the source code but ignore the execution environment, i.e. all the dependencies required to execute the software (including the OS) and their configurations, e.g., software repositories such as GitHub [9], Bitbucket [4], and RunMyCode [17]. And others, store the environments as a Virtual Machine (VM) image that lacks the detail that source code provides, e.g. Reprozip [16], CDE [6], and Umbrella [11].

In this paper, we show how the former do not provide enough detail about the artifact being executed, and how neither of these approaches provide enough detail about the environment running the artifacts. As such, more complex and self-contained tools are needed, tools that respect both the preservation of software artifact that contains or generates data and the long-term ability to inspect and execute them. In addition to preserving the ability of replicating past results, such a tool must also preserve the ability to discover errors in the software and update that software. Only then can the software stay relevant as new bugs are discovered and corrected. We also discuss how such a tool should support long-term reproducibility efforts by other scientists, and describe how we developed Open Curation for Computational Artifact Management (Occam) [14] (released on a GNU AGPLv3 license) towards solving these problems by providing a mechanism to create an on-demand environment to execute each application, supported by a software repository, and by metadata rich enough to rebuild the execution environment for that software artifact from scratch should the need arise. Occam preserves all the steps of software experiments, from development, to deployment, and execution. It also encouraging transparent and accountable research with its openness, and the longevity of its experiments, by documenting the complete process. 


\section{REPEAT, REPLICATE, REPRODUCE}

There are multiple interpretations of the terms around obtaining the same results: repeatability, replicability, and reproducibility. In this work we use the ACM definitions [2]:

- Repeatability - Authors can obtain their results multiple times using their artifacts

- Replicability - A different team can obtain the authors' results using their artifacts

- Reproducibility - A different team can obtain the authors' results using independently-developed artifacts

At first, the entry-level repeatability seems easy to guarantee, just preserve all the source code using a platform such as [7]. But within a few weeks from code implementation, few developers in fact remember exactly how they ran the experiments. As such, simple tools such as ReDoc [18] proposes to use Makefile-based scripts as a solution for this problem. Therefore, as long as the environment is preserved, and build/run scripts for all the execution steps are preserved with the code, then results can be easily repeated.

The second level, replicability, is more challenging, as anyone who has tried to compile software can attest to. One of the common issues at this level is the lack of detail in the artifact's documentation, in particular its dependencies. In order to re-compile and re-run code in a different machine and replicate the results, one must be able to rebuild all elements in the environment. However, even when authors are aware of this requirement and try to document all dependencies as completely as possible, there are unexpected issues that may arise. Examples abound, a few documented examples follow. In [13], while trying to replicate some of their previous results obtained using their own methodology for reproducibility, the authors struggled with dependencies. And in [10], a change in how glibc handles floating point numbers lead to significantly different results.

Some solutions to this problem were proposed. One is to detect files in the environment that are required in the execution, and package them together with the artifact itself $[6,16]$. Another solution is to pack the binaries from different dependencies and assembling them in a new environment [11], but not necessarily preserving the source code and the ability to rebuild it. However, these solutions focus on the immediate need for result replication, not for long-term reproducibility. Preserving the ability of running the exact same experiment, albeit essential, is not enough to guarantee reproducibility in the long run. Moreover, the inability to recreate binaries does not practically allow corrections of errors detected after the fact.

Lastly, designing software that can be useful for reproducibility is much more difficult and challenging. According to the ACM definition, reproducing an experiment requires a different team to implement someone else's software and successfully obtain the same results. If the effort is successful, then there is additional evidence that the original work is correct; however, if the reproduction fails, the only conclusion is that there is an error somewhere (Figure 1). We must be able to answer the following questions about the experimental results :

(1) What artifact produced this result?

(2) Can I inspect the artifact's source code?

(3) What are its run/build dependencies?

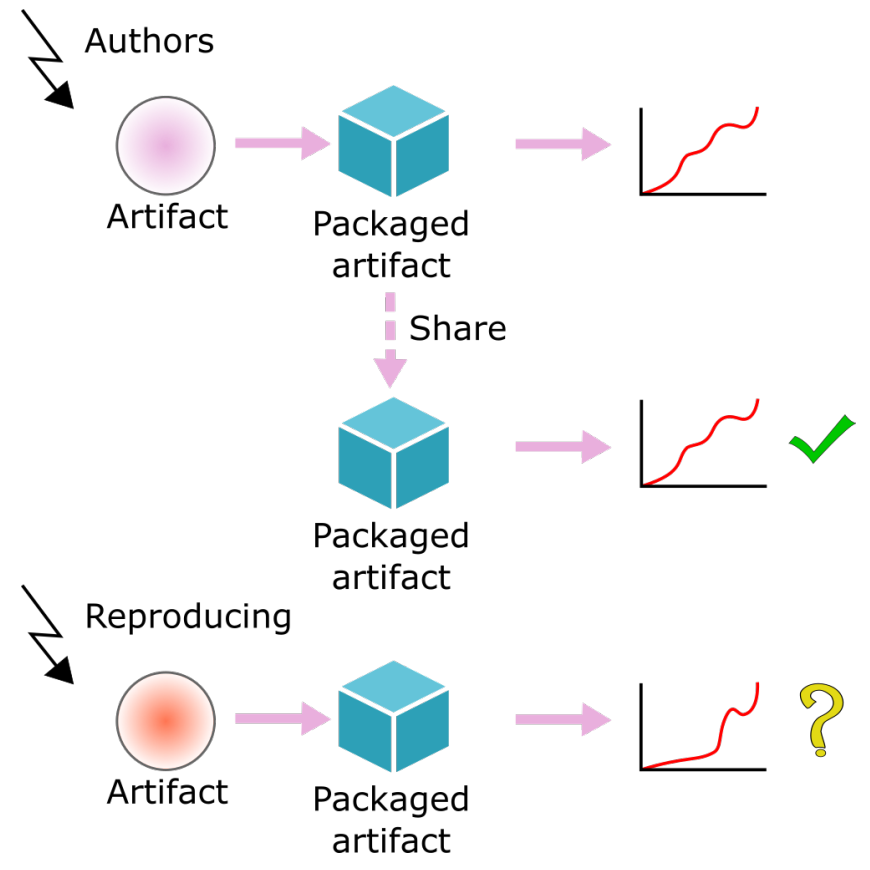

Figure 1: Failure to reproduce results only implies there is an error somewhere, not where that error is.

(4) Can I answer questions (2)-(4) for each of its dependencies?

In order to find the source of that error, and errors are ubiquitous [8], scientists must be able to inspect the original experiment, and compare implementations to find which part of the program is flawed. Furthermore, to repair the error, they may have to inspect the conditions under which software was built. They would have to, for instance, determine if the error is in one of its dependencies, compare build flags, and compare execution options. Moreover, when that error is found and corrected, the usefulness of repeating old experiments (with errors) is limited, when compared with running them error free. As such, we argue that packaging binaries is no longer enough. To have truly future-proof reproducible experiments, the source of the complete environment must be preserved.

\section{THE REQUIREMENTS FOR LONG-TERM REPRODUCIBILITY}

It is not easy to predict if what we currently preserve is enough to guarantee future execution. In fact, this is a very complex problem that affects (and brings together) fields as different as Humanities and Computer Science. For example, if you preserve your documents as a PDF but don't preserve the software that reads that format, what guarantees that you will be able to read that file format in 5-10 years, or even 50 years? Similarly, and more generally, how can one guarantee that executing software within the same time frame will yield the same results?

\subsection{Preserving all sources}

As we previously explained, preserving the complete execution environment is essential for providing software that can be used 


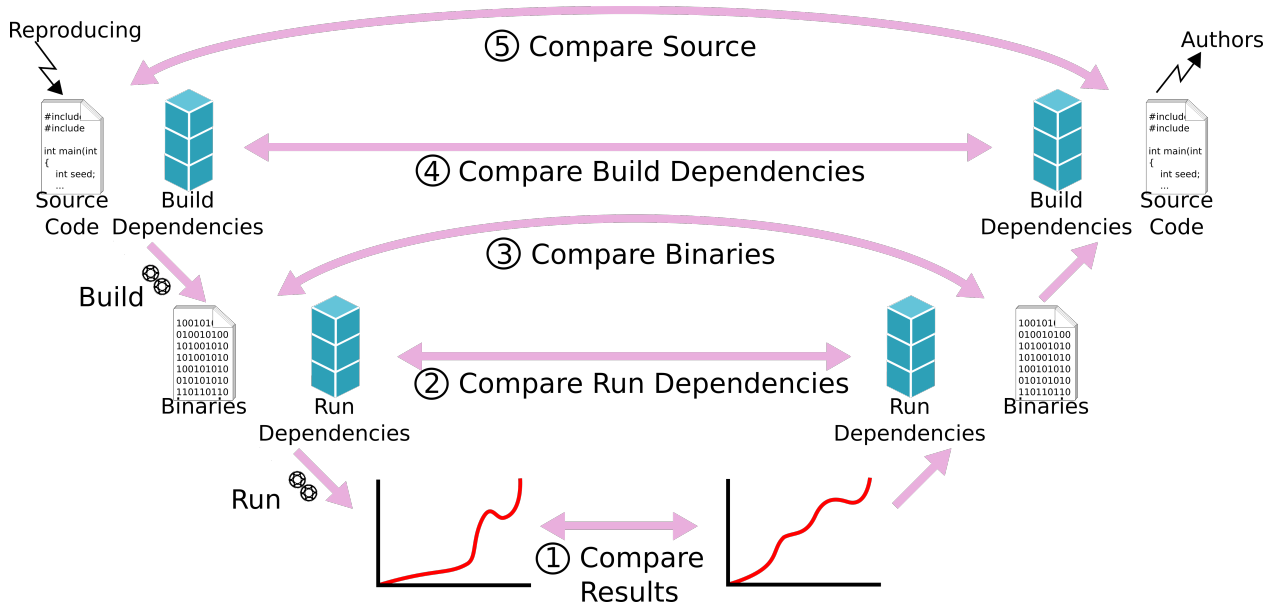

Figure 2: Depiction of the process of finding errors after a failed reproduction attempt.

for reproducible research. In fact, the more we preserve, the higher the likelihood of being able to replicate and reproduce past results, or to find the source of discrepancies. For example, in Figure 2 we see a depiction of the process of finding errors after a failed reproduction attempt. The team attempting the reproduction (left) obtains results to compare with the original results (1). Then, until the error is found, they can climb up the ladder comparing the different elements in the chain: (2) run dependencies, (3) binaries, (4) build dependencies, (5) the source code. Only if every dependency is itself a fully preserved artifact, can the right side of the ladder be fully inspected. Therefore, preserving the sources of dependencies is as important as preserving the sources of the artifact itself.

By preserving only binaries, this is where most of available platforms are currently lacking. Some frameworks, such as CDE [6] and ReproZip [16], only capture a subset of the environment; this is accomplished by instrumenting the binaries and log system-calls to find which files in the system are necessary for the execution, and then by packaging those files. This approach accomplishes the requirements for replicability, as those archives can be re-deployed and re-executed. However, reproducibility is hindered, as it requires independent verification including gathering independent input datasets. However, since these artifacts contain only parts of the original software, running new experiments for comparison purposes becomes potentially unpredictable. On the other hand, Umbrella [11] packages base environments (e.g., simple Linux images), and packages other binaries in such a way that they can be assembled into those execution environments; while this presents a more flexible approach, as all binaries belonging to an artifact are available, the source code and the ability to build it is not preserved. A similar, albeit less flexible, approach is taken by Pachyderm [15] and Whole Tale [5], where a virtual machine (using Docker) is used to process input data and generate results. As a consequence, none of these frameworks were designed to preserve source code, and cannot be inspected past (3) on the ladder.

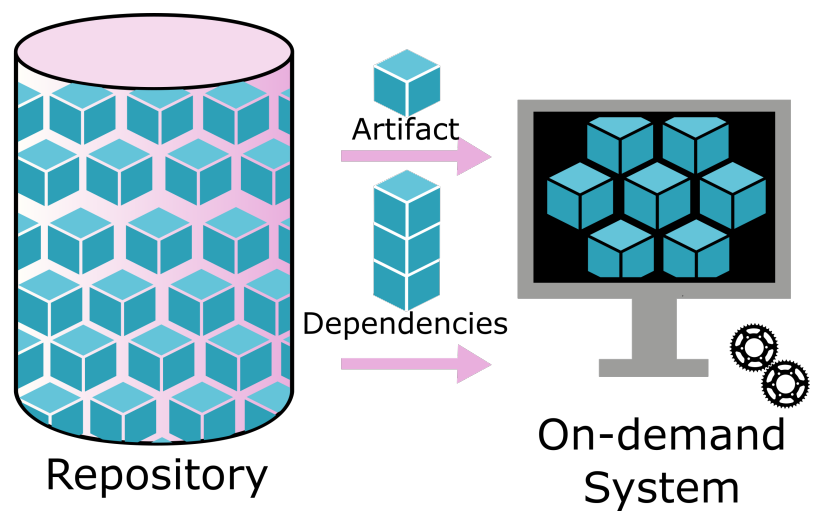

Figure 3: A framework that can build an OS on-demand from artifacts in a repository.

\subsection{Replicable and reproducible OSes}

Preserving complete execution environments in a monolithic package such as a compressed file or a VM image, despite preserving the ability of replicating past results, has a large number of disadvantages. The first is storage overhead. As previously discussed, approaches that only capture a small subset of the environment are not enough. Therefore, when we need to preserve the complete execution environments for every artifact (which may range from a few $\mathrm{MiB}$ for simple applications to multiple $\mathrm{GiB}$ for complex simulators), numbers quickly grow and storing VMs becomes problematic. Moreover, the software contained within those environments cannot be easily spliced and stitched into another environment, forcing the storage of many duplicates. This is especially problematic, as simple modifications (e.g., modifying one file) can lead to the duplication of the complete environment package.

The alternative is to preserve each element of the environment as an individual artifact, and store it in the repository. In fact, Umbrella [11] takes this approach. However, as their artifacts contain executable binaries, only, this is not enough. Preserving source code, and the ability to generate binaries from source is important to, as 
discussed before, finding/correcting bugs, and making the software useful in the future. Therefore, every element in the environment must be preserved as completely as any other artifact. Namely, build/run instructions must be provided, and all their software dependencies must be listed. Then the system can inspect the artifact description and automatically assemble the OS that it requires to execute, as depicted in Figure 3. Then, when an artifact executes, the system can find all of the required environment dependencies from the repository, create an OS using those dependencies, and execute it in an replicable environment. A big advantage of this approach, is that unless the artifact is not described properly, then the execution will fail from the start, thus if it is able to replication is ensured.

\section{SUPPORTING LONG TERM REPRODUCIBILITY WITH OCCAM}

Occam is an open curation platform that was built to support the execution of workflows representing complex software, such as computer architecture simulators. The focus of Occam is the preservation of software and its execution, and for that purpose it contains a set of services that can be used as the backbone of reproducible software execution.

\subsection{Occam overview}

Occam implements all of the elements required for the preservation of execution environments: a repository for artifact preservation, a software packaging schema that describes artifacts in detail, including dependencies, and their build and run instructions. This repository supports federated storage access, which facilitates the dissemination and access to artifacts, and it promotes artifact selfstorage and replication thus decreasing the likelihood of software being lost.

Due to Occam's initial focus on computer architecture simulation, Occam can support complex software artifacts. This includes software that has a large number of software dependencies, and that is often sensitive to changes in dependency versions. In particular, software that includes separate build and run dependencies (as we will explain hereinafter), which allows the overhead of large build times to be paid only once. In addition, Occam running environments are not preserved as mere monolithic VMs, instead they are built on-demand according to the requirements of each artifact. The fact that environments are built completely from other artifacts preserved by the system not only provides some guarantee to future users that the execution can, in fact, be replicated. But also that in case those users try to reproduce the results by implementing their own software and fail, they can inspect all the details of the original experiment and have the best chance to find the issue.

\subsection{Occam's artifacts}

Unlike other systems, where only the ability to run an artifact is preserved, Occam preserves all steps of software development. Occam's philosophy is that using software is not simply executing a binary and obtaining some results, therefore we cannot expect that storing binaries is enough to create reproducible experiments. Instead, the whole process of using software must be preserved:

(1) Code development

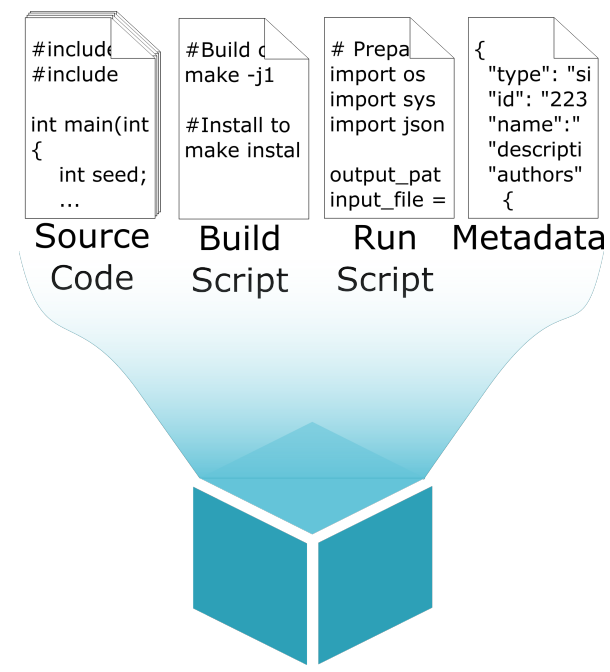

Figure 4: Depiction of a typical Occam object containing the source code, a build and run script, and metadata.

(2) Building the code

(3) Using the binaries

In order to accomplish this, the typical Occam package contains the source code, a build script, a run script, and metadata that describes the package (Figure 4). Listing 1 depicts a simplified metadata description of an Occam artifact. For the first step, Occam preserves the source code, identified by an Universally Unique Identifier (UUID), a type (e.g., simulator or CSV) that can be used by the system to type check experimental workflows (e.g., Occam checks the file types when the simulator requires a CSV file as input), and other human readable fields (such as name, description, and tags) that facilitate human interaction and discovery of artifacts. The artifact is managed by a git repository that tracks changes to the artifact and tracks revision numbers. Using the git repository allows for the inspection and execution of previous versions of the software. In order to preserve the second step, Occam explicitly support building artifacts as a step separate from running (thus allowing to build once, and run multiple times). Building an artifact requires declaring build time dependencies (i.e., the other artifacts in Occam that are needed to build this one), and a script that contains the build instructions (e.g., cflags, lflags, build commands). Optionally, external dependencies can be "installed" from the Internet; however, in order to preserve the reproducibility of the artifact, these are copied into Occam and preserved in the repository.

The final step, running the workflow, similarly to the building step, also has its own (different) dependencies and a run script containing the instructions/commands. Both building and running need to specify an architecture and environment where they can run (in the example, x86-64 on Linux). The environment itself is another artifact that provides the environment Linux on the required architecture. The artifact can also declare dependencies to other artifacts, for example libraries or tools it needs in various situations. 
Listing 1: Metadata of an Occam artifact

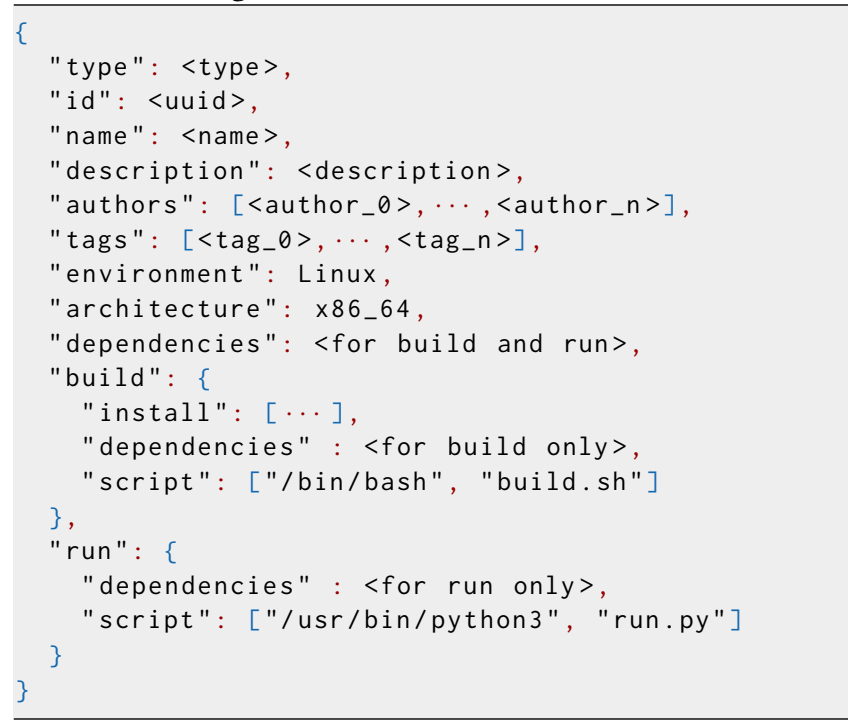

\subsection{Generating reproducible execution environments}

The most common approaches of preserving execution environments as virtual machines, or even of preserving packaged binaries are not enough to provide long-term usefulness of artifacts. They do not provide, specifically, the ability to inspect their execution, and they do not easily allow updates as bugs are discovered and improvements become available. As such, Occam implements a more complex, yet necessary, approach, depicted in Figure 5.

As previously explained, Occam artifacts contain source code, build/run instructions, and documented dependencies. Using this information, when the artifact needs to be built (Figure 5 left), Occam compiles a list of all required dependencies, and creates and stores a manifest in the repository. A manifest is a digest of the OS that is required to execute the artifact, i.e. a list of all artifacts (UUID and revision numbers) that are used to assemble an OS that can build the artifact (e.g., compilers, libraries, and tools) and the command that needs to be executed (i.e., how to invoke the build script). The results of the build operation (i.e., the built software) are stored in the repository cache to be used later; the built objects are only cached, as they can safely be deleted and rebuilt.

In a similar process (Figure 5 right), a manifest is also created to run an artifact. The only difference being the set of dependencies that are selected for the system, and the command to be executed (run script invocation). Results of a run (i.e. experimental results) are then automatically packaged in our repository format and added to the repository for future use. In this case, the results metadata includes a link to the manifest that was executed, allowing the result to be traced back the software that was used to create it.

Manifests are then translated into a set of instructions that create and execute that OS in an available execution backend. Occam currently supports Docker [12] - a lightweight OS-level virtualization software - and support for other system-level VM technologies are under development.

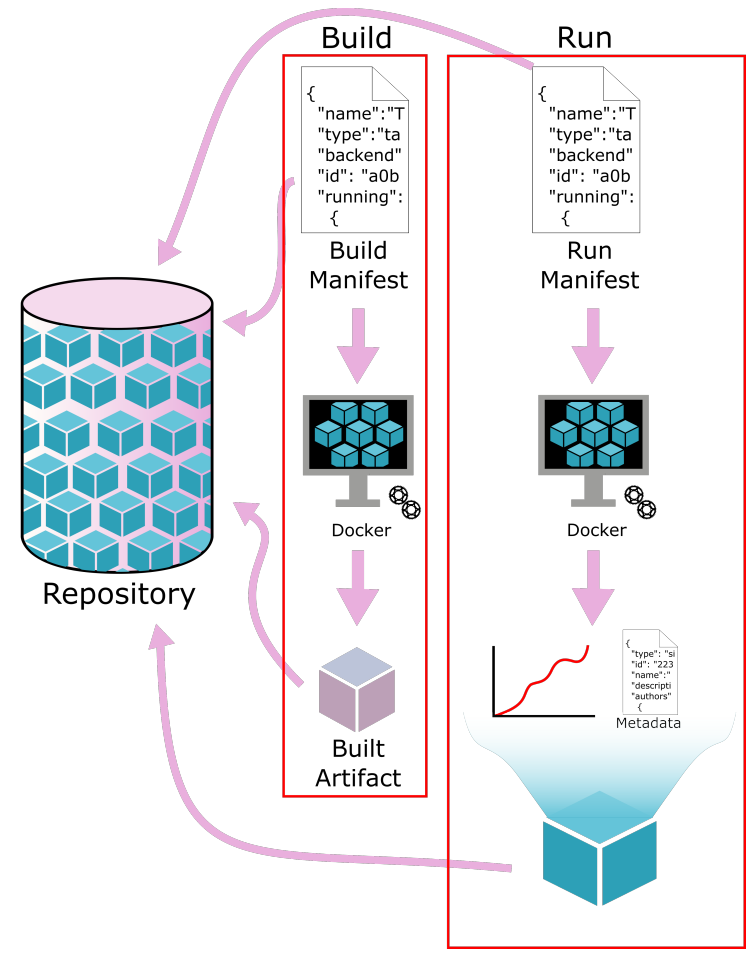

Figure 5: Depiction of Occam's artifact execution - (left) Building an artifact; (right) Running an artifact

\section{CONCLUSIONS}

Only time will tell us if a current curation effort will be enough to preserve software execution. However, the need to preserve as much of the experimental process as possible should be the aim of the scientific community.

In this position paper, we briefly discussed the current needs for long term reproducibility and how they can be supported by Occam, an open curation framework for computational artifacts. In essence, Occam preserves all software used in each execution, and the ability to re-build and re-run that software. Results are also kept in a repository, and linked to the software that created them for provenance, which facilitates the process of rooting out error that may exist.

Currently, we are developing the means to deploy manifests in system-level VMs, such as VirtualBox, and in native systems. As Docker is not enough for systems where experiments need to modify the OS (e.g., the Linux kernel), and some experiments may require to execute in an actual system.

\section{ACKNOWLEDGEMENTS}

The material in this document is based in part upon work supported by the National Science Foundation (NSF) under grant numbers ACI1535232 and CNS-1305220. Any opinions, findings, and conclusions or recommendations expressed in this material are those of the author(s) and do not necessarily reflect the views of the NSF. 


\section{REFERENCES}

[1] 2010. Reproducible Research. Computing in Science Engineering 12, 5 (Sept 2010), 8-13. https://doi.org/10.1109/MCSE.2010.113

[2] ACM. 2017. Artifact Review and Badging. http://www.acm.org/publications/ policies/artifact-review-badging. (2017).

[3] Monya Baker. 2016. 1,500 scientists lift the lid on reproducibility. Nature 533, 7604 (2016), 452-454.

[4] Bitbucket 2014. Bitbucket: Unlimited private code repositories. bitbucket.org. (2014). [Online; accessed 17-Nov-2014].

[5] Adam Brinckman, Kyle Chard, Niall Gaffney, Mihael Hategan, Matthew B. Jones, Kacper Kowalik, Sivakumar Kulasekaran, Bertram Ludäscher, Bryce D. Mecum, Jarek Nabrzyski, Victoria Stodden, Ian J. Taylor, Matthew J. Turk, and Kandace Turner. 2018. Computing environments for reproducibility: Capturing the "Whole Tale”. Future Generation Computer Systems (2018). https://doi.org/10.1016/j. future.2017.12.029

[6] cde 2017. CDE: Lightweight application virtualization for Linux. http://www. pgbovine.net/cde.html. (2017). [Online; accessed 29-Sep-2017].

[7] Roberto Di Cosmo and Stefano Zacchiroli. 2017. Software Heritage: Why and How to Preserve Software Source Code. In iPRES 2017: 14th International Conference on Digital Preservation (2017-0925). Kyoto, Japan. https://hal.archives-ouvertes.fr/hal-01590958https: //hal.archives-ouvertes.fr/hal-01590958/file/ipres-2017-software-heritage.pdf

[8] David L Donoho, Arian Maleki, Inam Ur Rahman, Morteza Shahram, and Victoria Stodden. 2009. Reproducible research in computational harmonic analysis. Computing in Science \& Engineering 11, 1 (2009), 8-18.

[9] GitHub 2014. GitHub: Build software better, together. github.com. (2014). [Online; accessed 12-Dec-2014].

[10] Tristan Glatard, Lindsay B. Lewis, Rafael Ferreira da Silva, Reza Adalat, Natacha Beck, Claude Lepage, Pierre Rioux, Marc-Etienne Rousseau, Tarek Sherif, Ewa Deelman, Najmeh Khalili-Mahani, and Alan C. Evans. 2015. Reproducibility of neuroimaging analyses across operating systems. Frontiers in Neuroinformatics 9 (2015), 12. https://doi.org/10.3389/fninf.2015.00012

[11] H. Meng, D. Thain, A. Vyushkov, M. Wolf, and A. Woodard. 2016. Conducting reproducible research with Umbrella: Tracking, creating, and preserving execution environments. In 2016 IEEE 12th International Conference on e-Science (e-Science). 91-100. https://doi.org/10.1109/eScience.2016.7870889

[12] Dirk Merkel. 2014. Docker: Lightweight Linux Containers for Consistent Development and Deployment. Linux J. 2014, 239, Article 2 (March 2014). http://dl.acm.org/citation.cfm?id=2600239.2600241

[13] Olivier Mesnard and Lorena A Barba. 2016. Reproducible and replicable CFD: it's harder than you think. arXiv preprint arXiv:1605.04339 (2016).

[14] Occam 2018. Occam - Open Curation for Computational Artifact Management. https://occam.cs.pitt.edu/. (2018). [Online; accessed 9-Apr-2018].

[15] Pachyderm 2018. Reproducible Data Science that Scales! - Pachyderm. https: //pachyderm.io. (2018). [Online; accessed 9-Apr-2018].

[16] ReproZip 2016. ReproZip - About. https://vida-nyu.github.io/reprozip/. (2016). [Online; accessed 29-Aug-2016].

[17] RunMyCode 2016. Run My Code. https://runmycode.org/. (2016). [Online; accessed 29-Aug-2016].

[18] Matthias Schwab, Martin Karrenbach, and Jon Claerbout. 2000. Making scientific computations reproducible. Computing in Science \& Engineering 2, 6 (2000), 61-67.

[19] et al. S.J. Hettrick. 2014. UK Research Software Survey 2014. (2014). https: //doi.org/DOI:10.5281/zenodo.1183562

[20] Victoria Stodden, Marcia McNutt, David H. Bailey, Ewa Deelman, Yolanda Gil, Brooks Hanson, Michael A. Heroux, John P.A. Ioannidis, and Michela Taufer. 2016. Enhancing reproducibility for computational methods. Science 354, 6317 (2016), 1240-1241. https://doi.org/10.1126/science.aah6168 arXiv:http://science.sciencemag.org/content/354/6317/1240.full.pdf

[21] David Wilkinson, Luis Oliveira, Bruce Childers, and Daniel Mosse. 2017. Evaluating Interactive Archives. (Oct 2017). https://doi.org/10.6084/m9.figshare.5483836. v1 